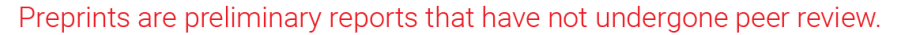 They should not be considered conclusive, used to inform clinical practice, or referenced by the media as validated information. \\ Spatial Similarity of MRI-Visible Perivascular Spaces in Healthy Young Adult Twins
}

\section{Boeun Lee}

Ewha Womans University, Ewha Womans University Seoul Hospital

Na-Young Shin ( $\square$ nyshin@catholic.ac.kr)

The Catholic University of Korea

\section{Chang-hyun Park}

The Catholic University of Korea

\section{Yoonho Nam}

Hankuk University of Foreign Studies

\section{Soo Mee Lim}

Ewha Womans University, Ewha Womans University Seoul Hospital

Kook Jin Ahn

The Catholic University of Korea

\section{Research Article}

Keywords: Brain perivascular space, Virchow-Robin Space, Location, Similarity, Twin Study

Posted Date: November 11th, 2021

DOI: https://doi.org/10.21203/rs.3.rs-1052220/v1

License: (c) (1) This work is licensed under a Creative Commons Attribution 4.0 International License.

Read Full License 


\section{Abstract \\ Purpose}

This study aims to determine whether genetic factors affect the location of dilated perivascular spaces (dPVS) by comparing healthy young twins and non-twin (NT) siblings.

\section{Methods}

A total of 700 healthy young adult twins and NT siblings (138 monozygotic (MZ) twin pairs, 79 dizygotic (DZ) twin pairs, and 133 NT sibling pairs) were collected from the Human Connectome Project dataset. dPVS was automatically segmented and normalized to standard space. Then, spatial similarity indices (mean squared error [MSE], structural similarity [SSIM], and dice similarity [DS]) were calculated for dPVS in the basal ganglia (BGdPVS) and white matter (WMdPVS) between paired subjects before and after propensity score matching of dPVS volumes between groups. Within-pair correlations for the regional volumes of dVPS were also assessed using the intraclass correlation coefficient (ICC).

\section{Results}

The spatial similarity of dPVS was significantly higher in MZ twins (higher DS [median, 0.382 and 0.310 ] and SSIM [0.963 and 0.887] and lower MSE [0.005 and 0.005] for BGdPVS and WMdPVS, respectively) than DZ twins (DS [0.121 and 0.119], SSIM [0.941 and 0.868], and MSE [0.010 and 0.011]) and NT siblings (DS [0.106 and 0.097], SSIM [0.924 and 0.848], and MSE [0.016 and 0.017]). No significant difference was found between DZ twins and NT siblings. Similar results were found even after subjects were matched according to dPVS volume. Regional dPVS volumes were also more correlated within pairs in MZ twins than DZ twins and NT siblings.

\section{Conclusion}

Our results suggest that genetic factors affect the location of dPVS.

\section{Introduction}

Cerebral perivascular spaces (PVS) are cerebrospinal fluid (CSF)-filled structures that wrap around the arteries, arterioles, veins, and venules as the vessels enter and exit the brain. After their structure was first described by Virchow and Robin, much research was done to further understand their anatomy, function and dysfunction ${ }^{1}$. Recently, PVS were found to belong to the glymphatic system, a recently discovered macroscopic waste clearance system, and to function as part of waste cleaning, energy substrate delivery and blood flow regulation ${ }^{2}$ as well as act as an early imaging marker for cerebral small vessel disease ${ }^{3-}$ 
5. PVS may be dilated and visible on MRI under certain situations, such as aging, cerebral small vessel disease ${ }^{3}$, hypertension ${ }^{6}$, intracranial hemorrhage ${ }^{7}$, multiple sclerosis ${ }^{8,9}$, Alzheimer's disease ${ }^{10}$, and Parkinson disease. ${ }^{11}$

The exact mechanism of this dilatation of PVS is still unclear, but probably influenced by both genetic and environmental factors. ${ }^{12}$. A study with a large elderly population found that dilated PVS (dPVS) burden assessed with a 4-point visual scale was highly heritable ${ }^{13}$. Another recent study with a healthy young population replicated this result by showing that dPVS volume measured using an automated segmentation method was highly heritable as well ${ }^{14}$. Both studies consistently found dPVS in white matter (WMdPVS) to be a more heritable trait than dPVS in the basal ganglia (BGdPVS), suggesting a distinct genetic impact on dPVS burden according to regional location. However, we do not yet know if the location of each dPVS itself is also genetically determined.

Therefore, in this study, we aimed to evaluate whether dPVS location is influenced by genetic factors by comparing spatial similarity indices in healthy young adult twins and non-twin (NT) siblings.

\section{Materials And Methods}

This retrospective study was approved by our institutional review board (The Catholic University of Korea Seoul St. Mary's Hospital). The requirement for informed consent was waived as we used a publicly available dataset for this study. The methods and reporting of results are in accordance with the STROBE (Strengthening the Reporting of Observational Studies in Epidemiology) guidelines.

\subsection{Study Population}

dPVS were segmented using structural images and demographic information obtained from the WU-Minn Human Connectome Project dataset which enrolled healthy adult twins and NT siblings between the ages of 22 to 35 to identify relationships between brain circuits, genetics and behavior ${ }^{15}$.

Of 1206 subjects included in the March 2017 (S1200) release, 1113 subjects who underwent 3T MRI to obtain $0.7 \mathrm{~mm}$ isotropic 3D T1- and T2-weighted images were initially enrolled. The exclusion criteria were as follows: high blood pressure, diabetes mellitus or significant cardiovascular disease; severe neurodevelopmental, neurological or documented neuropsychiatric disorders; zygosity not examined by genotyping; NT siblings without respective pairs; and birth before 34th weeks of gestation for twins and before 37 weeks of gestation for non-twins. More information on recruitment and the inclusion and exclusion criteria of the Human Connectome Project was described in a previous study ${ }^{15}$

\subsection{Imaging acquisition}

All MRI data were acquired using a 3T MR scanner (MAGNETOM Skyra CONNECTOM, Siemens Healthcare) customized with a $100 \mathrm{mT} / \mathrm{m}$ gradient coil, inner bore diameter of $56 \mathrm{~cm}$, and a standard $32-$ channel head coil at Washington University in St. Louis, MO, USA. 
The 3D T1-weighted Magnetization-Prepared Rapid Acquisition with Gradient Echo (MPRAGE) sequence was performed using the following parameters: sagittal acquisition with repetition time, $2400 \mathrm{~ms}$; echo time, $2.14 \mathrm{~ms}$; field of view, $224 \times 224 \times 180 \mathrm{~mm}$; voxel size, $0.7 \times 0.7 \times 0.7 \mathrm{~mm}^{3}$; inversion time, $1000 \mathrm{~ms}$; band width, $210 \mathrm{~Hz} /$ pixel; flip angle, $8^{\circ}$; GeneRalized Autocalibrating Partial Parallel Acquisition (GRAPPA) factor, 2; and total acquisition time, 7 min 40 s.

The 3D T2-weighted Sampling Perfection with Application-optimized Contrasts using different flip angle Evolution (SPACE) sequence was performed using the following parameters: sagittal acquisition with repetition time, $3200 \mathrm{~ms}$; echo time, 565 ms; echo spacing, 3.53 ms; turbo factor, 314; echo train duration , $1105 \mathrm{~ms}$; field of view, $224 \times 224 \times 180 \mathrm{~mm}$; voxel size $=0.7 \times 0.7 \times 0.7 \mathrm{~mm}^{3}$; band width, $744 \mathrm{~Hz} / \mathrm{pixel}$; variable flip angle; GRAPPA factor, 2; and total acquisition time, 8 min 24s.

More details on the imaging protocols are described in the WU-Minn Human Connectome Project S1200 Release Reference Manual 15

\subsection{Spatial similarity assessment}

The segmentation of dPVS was a fully automated process that was described in detail in a previous study ${ }^{14}$. It entailed the extraction of potential voxels for dPVS using a 3D Frangi filter after signal normalization of 3D T2-weighted images. To reduce false positives outside the brain parenchyma, potential dPVS voxels only inside the BG and WM masks of the Freesurfer segmentation were selected. In addition, we trained and applied a 3D deep convolutional neural network to distinguish dPVS from the false-positive voxels. Based on the final output of the 3D deep learning algorithm, dPVS masks for each BG and WM were obtained.

To compare dPVS locations between pairs, we assessed the similarity of their dPVS images. T1-weighted images on which dPVS had been defined were used to first estimate a deformation field as it was needed for the spatial transformation between each subject's brain images and the template brain images in SPM12 (https://www.fil.ion.ucl.ac.uk/spm/software/spm12/). The deformation field was then used to transform dPVS images to standard space, and the locations of dPVS in the standard space were compared using the following three similarity indices: mean squared error (MSE), structural similarity (SSIM), and dice similarity (DS). MSE and SSIM have been proposed as metrics for image quality assessment in prior studies. While MSE is a metric simply computed by averaging the squared intensity differences between two images, SSIM is a metric for comparing local patterns of intensities ${ }^{16}$. DS is a metric for gauging the similarity of two sets based on their cardinalities ${ }^{17}$, and can be applied to two binary images to assess the commonality between them with values ranging between 0 and 1 . We compared the locations of dPVS separately for BG and WM.

\subsection{Cognitive function assessment}

The well-validated NIH Toolbox Cognition Battery was used to assess cognitive function ${ }^{18}$. The Battery contains subtests that assess five cognitive domains: executive function (Dimensional Change Card Sort 
Test [cognitive flexibility], Flanker Inhibitory Control and Attention Test [inhibitory control and attention]), processing speed (Pattern Comparison Processing Speed Test), working memory (List Sorting Working Memory Test), episodic memory (Picture Sequence Memory Test), and language (Picture Vocabulary Test [vocabulary], Oral Reading Recognition Test [reading decoding]).

\subsection{Statistical Analysis}

After normality tests were performed, age, brain regional and dPVS volumes, and all similarity indices were compared between the three groups using the Kruskal-Wallis test and then the post-hoc Dunn's test with Bonferroni adjustment. The frequency of sex was compared between the groups using the chisquared test with Bonferroni adjustment.

As spatial similarity indices could be affected by the total volume of dPVS, we used propensity score matching separately for volumes of BGdPVS and WMdPVS to balance this confounding factor between groups using the nearest matching method with a 1:1:1 ratio ${ }^{19}$ The spatial similarity indices were also compared between groups in the matched subjects.

To define genetic influence on the regional location of dPVS, we first divided WM into four (i.e., frontal, parietal, temporal, and occipital) lobar subregions using the Freesurfer results available in the Human Connectome Project dataset. Then, we performed an intraclass correlation (ICC) analysis within twin or NT pairs for dPVS volumes in each of the BG and WM subregions. The ICC for twin data was calculated as:

\section{$I C C=\frac{\text { MSbetween }- \text { MSwithin }}{\text { MSbetween }+ \text { MSwithin }}$}

where $\mathrm{MS}_{\text {between }}$ and $\mathrm{MS}_{\text {within }}$ are the mean-square estimate of between- and within-pair variance, respectively 20 .

To assess the clinical implications of dPVS according to location, a correlation analysis was performed between regional dPVS burden and the cognitive function test results.

A $P$ value of $<0.05$ was considered statistically significant. All statistical analyses were performed using $\mathrm{R}$ Statistical Software (version 4.0.3; R Foundation for Statistical Computing, Vienna, Austria).

\section{Results}

\section{Demographics characteristics of the study population}

In this study, a total of 700 subjects including 138 monozygotic (MZ) twin pairs, 79 dizygotic (DZ) twin pairs, and 133 NT sibling pairs were finally included. The baseline characteristics of all participants are summarized in Table 1. Both MZ and DZ twins were older (mean age, 29 years old vs. 27 years old, $P<$ 
$0.001)$ and consisted of more male participants ( $58.7 \%$ and $62 \%$ vs. $46.2 \%, P=0.015$ and $P=0.007$, respectively) than NT siblings.

Table 1

Demographic and imaging characteristics of all participants

\begin{tabular}{|c|c|c|c|c|c|c|c|}
\hline & \multirow{2}{*}{$\begin{array}{l}\text { MZ twins } \\
(\mathrm{N}=276)\end{array}$} & \multirow{2}{*}{$\begin{array}{l}\text { DZ twins } \\
(\mathrm{N}=158)\end{array}$} & \multirow{2}{*}{$\begin{array}{l}\text { NT siblings } \\
(\mathrm{N}=266)\end{array}$} & \multirow{2}{*}{$\begin{array}{l}P \\
\text { value }\end{array}$} & \multicolumn{3}{|c|}{ Post hoc analysis ${ }^{a}$} \\
\hline & & & & & $P 1$ & $P 2$ & $P 3$ \\
\hline Age & $\begin{array}{l}29.0(27.0- \\
32.0)\end{array}$ & $\begin{array}{l}29.0(27.0- \\
32.0)\end{array}$ & $\begin{array}{l}27.0(24.0- \\
31.0)\end{array}$ & $<0.001$ & 1.000 & $<0.001$ & $<0.001$ \\
\hline Sex, male & $162(58.7 \%)$ & $98(62.0 \%)$ & $123(46.2 \%)$ & 0.002 & 1.000 & 0.015 & 0.007 \\
\hline $\mathrm{BG}\left(\mathrm{cm}^{3}\right)$ & $\begin{array}{l}22.3(21.1- \\
24.1)\end{array}$ & $\begin{array}{l}22.7(21.2- \\
24.7)\end{array}$ & $\begin{array}{l}23.3(21.7- \\
24.7)\end{array}$ & 0.001 & 0.266 & $<0.001$ & 0.482 \\
\hline \multirow[t]{2}{*}{$\mathrm{WM}\left(\mathrm{cm}^{3}\right)$} & \multirow{2}{*}{$\begin{array}{l}434.4 \\
(405.1- \\
474.1)\end{array}$} & 437.6 & \multirow{2}{*}{$\begin{array}{l}448.9(404- \\
485.3)\end{array}$} & \multirow[t]{2}{*}{0.193} & & & \\
\hline & & $\begin{array}{l}(408.5- \\
480.1)\end{array}$ & & & & & \\
\hline $\mathrm{ICV}\left(\mathrm{cm}^{3}\right)$ & $\begin{array}{l}1589.2 \\
(1459.8- \\
1695.2)\end{array}$ & $\begin{array}{l}1554.9 \\
(1471.6- \\
1718.3)\end{array}$ & $\begin{array}{l}1607.5 \\
(1486.4- \\
1742)\end{array}$ & 0.081 & & & \\
\hline $\begin{array}{l}\text { BGdPVS } \\
\left(\mathrm{cm}^{3}\right)\end{array}$ & $\begin{array}{l}0.229 \\
(0.198- \\
0.285)\end{array}$ & $\begin{array}{l}0.240(0.207- \\
0.294)\end{array}$ & $\begin{array}{l}0.225 \\
(0.184- \\
0.265)\end{array}$ & 0.001 & 0.138 & 0.119 & $<0.001$ \\
\hline $\begin{array}{l}\text { WMdPVS } \\
\left(\mathrm{cm}^{3}\right)\end{array}$ & $\begin{array}{l}1.951 \\
(1.448- \\
2.717)\end{array}$ & $\begin{array}{l}1.918(1.405- \\
2.431)\end{array}$ & $\begin{array}{l}1.744 \\
(1.314- \\
2.400)\end{array}$ & 0.022 & 1.000 & 0.021 & 0.223 \\
\hline
\end{tabular}

Data are expressed as medians with interquartile ranges or as numbers with percentages in parentheses.

a $P 1: M Z$ twin vs. DZ twin, $P 2$ : $M Z$ twin vs. non-twin sibling, $P 3$ : $D Z$ twin vs. non-twin sibling Abbreviations: BG, basal ganglia; BGdPVS, dilated perivascular space in basal ganglia; DZ, dizygotic; ICV, intracranial volume; MZ, monozygotic; NT, non-twin; WM, white matter; WMdPVS, dilated perivascular space in white matter.

BGdPVS volumes were greater in DZ twins than in NT siblings $\left(0.240 \mathrm{~cm}^{3}\right.$ vs. $\left.0.225 \mathrm{~cm}^{3}, P<0.001\right)$ while WMdPVS volumes were greater in MZ twins than in NT siblings $\left(1.951 \mathrm{~cm}^{3}\right.$ vs. $\left.1.744 \mathrm{~cm}^{3}, P=0.021\right)$. No significant differences were found between the three groups for other characteristics.

\section{Group Comparisons Of Spatial Similarity Indices In All Subjects}


All spatial similarity indices were different among the three groups for both BGdPVS and WMdPVS (Table 2). MZ twins showed higher spatial similarity-higher DS (0.382) and SSIM (0.963) and lower MSE (0.005) -for BGdPVS compared to DZ twins (DS, 0.121, $P<.001$; SSIM, 0.941, $P=.015$; MSE, 0.010, $P$ $=.004$ ) and NT siblings (DS, 0.106; SSIM, 0.924; MSE, 0.016, all $P S<.001)$, respectively. The spatial similarity of WMdPVS was also higher in MZ twins (DS, 0.310; SSIM, 0.887; MSE, 0.005) compared to DZ twins (DS, 0.119, $P<.001 ;$ SSIM, 0.868, $P=.014$; MSE, 0.011, $P=.004$ ) and NT siblings (DS, 0.097; SSIM, 0.848; MSE, 0.017, all $P S<.001)$. There was no significant difference in spatial similarity indices between the DZ and NT groups for both BGdPVS and WMdPVS. There were more overlapping dPVS voxels between paired subjects across all subjects in MZ twins than DZ twins and NT siblings (Fig. 1).

Table 2

Comparison of spatial similarity indices between the three groups in all subjects

\begin{tabular}{|c|c|c|c|c|c|c|c|c|}
\hline & & $\begin{array}{l}\text { MZ twin } \\
\text { pairs }\end{array}$ & $\begin{array}{l}\text { DZ twin } \\
\text { pairs }\end{array}$ & $\begin{array}{l}\text { NT sibling } \\
\text { pairs }\end{array}$ & $\begin{array}{l}P \\
\text { value }\end{array}$ & Post ho & analysis & \\
\hline & & $(n=138)$ & $(n=79)$ & $(n=133)$ & & $P 1$ & $P 2$ & $P 3$ \\
\hline BGdPVS & DS & $\begin{array}{l}0.382 \\
(0.113- \\
0.643)\end{array}$ & $\begin{array}{l}0.121 \\
(0.012- \\
0.314)\end{array}$ & $\begin{array}{l}0.106 \\
(0.015- \\
0.311)\end{array}$ & $<0.001$ & $<0.001$ & $<0.001$ & 1.000 \\
\hline & SSIM & $\begin{array}{l}0.963 \\
(0.927- \\
0.987)\end{array}$ & $\begin{array}{l}0.941 \\
(0.892- \\
0.979)\end{array}$ & $\begin{array}{l}0.924 \\
(0.870- \\
0.968)\end{array}$ & $<0.001$ & 0.015 & $<0.001$ & 0.081 \\
\hline & MSE & $\begin{array}{l}0.005 \\
(0.001- \\
0.013)\end{array}$ & $\begin{array}{l}0.010 \\
(0.002- \\
0.028)\end{array}$ & $\begin{array}{l}0.016 \\
(0.004- \\
0.033)\end{array}$ & $<0.001$ & 0.004 & $<0.001$ & 0.156 \\
\hline WMdPVS & DS & $\begin{array}{l}0.310 \\
(0.037- \\
0.605)\end{array}$ & $\begin{array}{l}0.119 \\
(0.012- \\
0.248)\end{array}$ & $\begin{array}{l}0.097 \\
(0.015- \\
0.286)\end{array}$ & $<0.001$ & $<0.001$ & $<0.001$ & 1.000 \\
\hline & SSIM & $\begin{array}{l}0.887 \\
(0.848- \\
0.916)\end{array}$ & $\begin{array}{l}0.868 \\
(0.809- \\
0.899)\end{array}$ & $\begin{array}{l}0.848 \\
(0.787- \\
0.900)\end{array}$ & $<0.001$ & 0.014 & $<0.001$ & 0.693 \\
\hline & MSE & $\begin{array}{l}0.005 \\
(0.002- \\
0.014)\end{array}$ & $\begin{array}{l}0.011 \\
(0.003- \\
0.029)\end{array}$ & $\begin{array}{l}0.017 \\
(0.005- \\
0.034)\end{array}$ & $<0.001$ & 0.004 & $<0.001$ & 0.1950 \\
\hline
\end{tabular}

Data are expressed as medians with interquartile ranges.

a $P 1$ : $M Z$ twin vs. DZ twin, $P 2$ : $M Z$ twin vs. non-twin sibling, $P 3$ : DZ twin vs. non-twin sibling

Abbreviations: BG, basal ganglia; BGdPVS, dilated perivascular space in basal ganglia; DS, dice similarity; DZ, dizygotic; MSE, mean squared error; MZ, monozygotic; NT, non-twin; SSIM, structural similarity; WM, white matter; WMdPVS, dilated perivascular space in white matter. 
Higher volumes of dPVS might result in spuriously higher spatial similarity due to the overlapping of adjacent dPVSs. Therefore, to avoid this possibility, we matched dPVS volumes between the groups for BGdPVS and WMdPVS separately. 72 subjects were selected from each group for BGdPVS and 51 subjects were selected from each group for WMdPVS. Spatial similarity indices still indicated that dPVS were most similarly located within the MZ twin pairs for both BGdPVS and WMdPVS, although some of the similarity indices no longer showed statistical significance (Table 3).

Table 3

Comparison of spatial similarity indices between the three groups in subjects matched for dPVS volumes

\begin{tabular}{|c|c|c|c|c|c|c|c|c|}
\hline & & \multirow{2}{*}{$\begin{array}{l}\text { MZ twin } \\
\text { pairs }\end{array}$} & \multirow{2}{*}{$\begin{array}{l}\text { DZ twin } \\
\text { pairs }\end{array}$} & \multirow{2}{*}{$\begin{array}{l}\text { NT sibling } \\
\text { pairs }\end{array}$} & \multirow{2}{*}{$\begin{array}{l}P \\
\text { value }\end{array}$} & \multicolumn{3}{|c|}{ Post hoc analysis ${ }^{a}$} \\
\hline & & & & & & $P 1$ & $P 2$ & $P 3$ \\
\hline \multirow[t]{3}{*}{$\begin{array}{l}\text { BGdPVS } \\
(n=72)\end{array}$} & DS & $\begin{array}{l}0.398 \\
(0.115- \\
0.626)\end{array}$ & $\begin{array}{l}0.115 \\
(0.010- \\
0.272)\end{array}$ & $\begin{array}{l}0.134 \\
(0.039- \\
0.317)\end{array}$ & $<0.001$ & $<0.001$ & $<0.001$ & 1.000 \\
\hline & SSIM & $\begin{array}{l}0.957 \\
(0.926- \\
0.987)\end{array}$ & $\begin{array}{l}0.948 \\
(0.905- \\
0.980)\end{array}$ & $\begin{array}{l}0.926 \\
(0.869- \\
0.965)\end{array}$ & 0.001 & 0.324 & $<0.001$ & 0.069 \\
\hline & MSE & $\begin{array}{l}0.006 \\
(0.001- \\
0.013)\end{array}$ & $\begin{array}{l}0.009 \\
(0.002- \\
0.025)\end{array}$ & $\begin{array}{l}0.015 \\
(0.005- \\
0.030)\end{array}$ & $<0.001$ & 0.123 & $<0.001$ & 0.149 \\
\hline \multirow[t]{3}{*}{$\begin{array}{l}\text { WMdPVS } \\
(n=51)\end{array}$} & DS & $\begin{array}{l}0.271 \\
(0.040- \\
0.602)\end{array}$ & $\begin{array}{l}0.153 \\
(0.016- \\
0.248)\end{array}$ & $\begin{array}{l}0.078 \\
(0.015- \\
0.248)\end{array}$ & 0.002 & 0.015 & 0.003 & 1.000 \\
\hline & SSIM & $\begin{array}{l}0.891 \\
(0.844- \\
0.909)\end{array}$ & $\begin{array}{l}0.879 \\
(0.819- \\
0.903)\end{array}$ & $\begin{array}{l}0.861 \\
(0.810- \\
0.905)\end{array}$ & 0.060 & 0.382 & 0.058 & 1.000 \\
\hline & MSE & $\begin{array}{l}0.003 \\
(0.002- \\
0.013)\end{array}$ & $\begin{array}{l}0.011 \\
(0.002- \\
0.025)\end{array}$ & $\begin{array}{l}0.016 \\
(0.004- \\
0.032)\end{array}$ & 0.001 & 0.073 & $<0.001$ & 0.356 \\
\hline
\end{tabular}

Data are expressed as medians with interquartile ranges.

${ }^{a} P 1: M Z$ twin vs. DZ twin, $P 2: M Z$ twin vs. non-twin sibling, $P 3$ : DZ twin vs. non-twin sibling

Abbreviations: BG, basal ganglia; BGdPVS, dilated perivascular space in basal ganglia; DS, dice similarity; DZ, dizygotic; MSE, mean squared error; MZ, monozygotic; NT, non-twin; SSIM, structural similarity; WM, white matter; WMdPVS, dilated perivascular space in white matter.

\section{Within-pair Correlations For The Regional Volumes Of Dpvs}

MZ twin pairs showed higher ICCs for dPVS volumes than DZ twin pairs and NT sibling pairs regardless of the location of dPVS. In all regions, no significant difference was found between DZ twin pairs and NT 
sibling pairs (Table 4).

Table 4

Within-pair correlations for the regional volumes of dPVS

\begin{tabular}{|llll|}
\hline & $\begin{array}{l}\text { MZ twin pairs } \\
(\mathbf{n = 1 3 8 )}\end{array}$ & $\begin{array}{l}\text { DZ twin pairs } \\
(\mathbf{n = 7 9 )}\end{array}$ & $\begin{array}{l}\text { NT sibling pairs } \\
\mathbf{( n = 1 3 3 )}\end{array}$ \\
\hline BGdPVS & $0.691(0.660-0.720)$ & $0.278(0.206-0.347)$ & $0.263(0.207-0.316)$ \\
\hline Frontal WMdPVS & $0.896(0.884-0.907)$ & $0.327(0.257-0.393)$ & $0.292(0.238-0.345)$ \\
\hline Parietal WMdPVS & $0.867(0.852-0.881)$ & $0.348(0.279-0.413)$ & $0.268(0.212-0.321)$ \\
\hline Temporal WMdPVS & $0.808(0.787-0.827)$ & $0.281(0.209-0.350)$ & $0.297(0.242-0.349)$ \\
\hline Occipital WMdPVS & $0.604(0.566-0.639)$ & $0.256(0.183-0.326)$ & $0.311(0.257-0.363)$ \\
\hline Data are expressed as intraclass correlation coefficients with 95\% confidence intervals in parentheses. \\
\hline \multicolumn{2}{|l|}{$\begin{array}{l}\text { Abbreviations: BGdPVS, dilated perivascular space in basal ganglia; MZ, monozygotic; DZ, dizygotic; } \\
\text { NT, non-twin; WMdPVS, dilated perivascular space in white matter. }\end{array}$} \\
\hline \multicolumn{4}{|l}{ All correlations were significant with P<0.05. } \\
\hline
\end{tabular}

\section{Correlation analysis between regional volumes of dPVS and cognitive function}

There were no significant correlations between regional dPVS volumes and cognitive function test results (Table S1).

\section{Discussion}

This study evaluated whether the location of dPVS was affected by genetic factors using high quality images of healthy young twins and NT siblings from a large dataset. We demonstrated that the location of individual dPVS was most similar within pair members in MZ twins. The within-pair spatial similarity between DZ twins and NT siblings showed no significant difference. After matching dPVS volume, MZ twin pairs still showed the highest spatial similarity, although statistical significance did decrease. The regional volumes of dPVS were more highly correlated in MZ twin pairs than DZ twin pairs and NT sibling pairs, while the within-pair correlations of regional dPVS volumes were not significantly different between DZ twin pairs and NT sibling pairs. Therefore, our results suggest that genetics influence the location of dPVS.

Hitherto, a few studies have explored the heritability of dPVS burden in large populations. Duperron et al. found that dPVS burden was highly heritable in an elderly population ${ }^{7}$. Choi et al. replicated this result but in a healthy young adult population ${ }^{14}$. These two studies consistently reported WMdPVS as being more heritable than BGdPVS, suggesting that genetic contributions to dPVS burden differed by location. 
However, research on whether genetics affect the location of dPVS, instead of its burden, is lacking. A previous study assessed the burden of WM hyperintensities (WMH), which are a well-known imaging marker for cerebral small vessel disease ${ }^{3-5}$. The study reported WMH burden as being highly heritable for each of the cerebral lobes as well as for the whole brain ${ }^{21}$, suggesting that location as well as overall burden of WMH might be affected by genetic factors. Cerebral blood flow per each vascular territory, which is closely related with small vessel disease ${ }^{22,23}$, was also reported to be affected by genetic factors ${ }^{24}$. Therefore, although further study is needed, we can postulate that the location of dPVS as well as its overall burden might be genetically determined to some extent.

Knowledge on the location of dPVS might help predict clinical outcome. Previous studies have suggested distinct underlying pathophysiology and clinical manifestations of BGdPVS and WMdPVS. BGdPVS has been associated with aging, hypertension ${ }^{25}$, small vessel disease including $\mathrm{WMH}$, lacunar infarction, deep microbleeds $5,13,25,26$, and subcortical vascular cognitive impairment ${ }^{10}$. In comparison, WMdPVS has been associated with the apolipoprotein $E \varepsilon 4$ allele, cortical amyloid- $\beta$ deposition ${ }^{10,27}$, cerebral amyloid angiopathy with cortical superficial siderosis, lobar microbleeds, and lobar hemorrhage ${ }^{25,28}$, and Alzheimer's disease ${ }^{10}$. Recent longitudinal studies have even shown distinct associations between dPVS and future dementia risk ${ }^{29-31}$, recurrent ischemic ${ }^{32}$ and hemorrhagic stroke ${ }^{33}$, and progressive deterioration of cognitive and motor symptoms in Parkinson's disease ${ }^{11,34}$ according to the location of PVS. Besides the distinction between BGdPVS and WMdPVS, decreased neuronal and axonal densities with reactive gliosis adjacent to dPVS ${ }^{35}$ and increased CSF markers of neurodegeneration associated with higher burden of dPVS in past studies suggest that a specific location of dPVS might cause differential clinical outcomes just as the lobar location of WMH differentially affects cognition and behavior ${ }^{36,37}$. However, in our study, we failed to find any significant associations between regional dPVS volumes and specific cognitive functions. This might be because our study population was made up of healthy young adults whose normal range of cognition is not broad enough to sufficiently show any significant correlation with dPVS volume. Another possible explanation might be that dPVS is an early imaging marker for small vessel disease or neurodegeneration, so, clinical manifestations of dPVS might not develop by the date of the MRI. To elucidate differential clinical outcomes per dPVS location, a longitudinal study on a large population with a wide range of cognitive and behavioral characteristics should be conducted.

There were several limitations to this study. First, this study was performed retrospectively with publicly available data. Therefore, longitudinal follow-up data was not available for the study population, which limits our understanding of the differential clinical implications of dPVS according to location. Further longitudinal study is required. Second, the similarity measurement methods of this study are not yet regularly used to compare medical images between different subjects and thus, the reliability of the similarity indices has not yet been sufficiently demonstrated. Third, although propensity score matching can help balance the volume differences between the three groups, biases caused by yet unknown factors might still remain. 


\section{Conclusion}

Our study found evidence that genetics are one deciding factor of dPVS location.

\section{Declarations}

\section{Availability of data and material}

Data are only available upon request, and before the request, data cannot be shared publicly by the regulation of Institutional Review Board of The Catholic University of Korea Seoul St. Mary's Hospital, because data may contain potentially identifying or sensitive patient information.

\section{Authors' contributions}

Boeun Lee, MD, Na-Young Shin, MD, PhD, Chang-hyun Park, PhD, Yoonho Nam, PhD, Soo Mee Lim, MD, PhD, Kook Jin Ahn, MD, PhD

Conception or design of the work: N.Y.S.

Acquisition, analysis, and interpretation of data for the work: B.L., N.Y.S., C.H.P., Y.N., S.M.L., K.J.A.

Drafting the work: B.L., N.Y.S.

Revising the work critically for important intellectual content: B.L., N.Y.S.

Final approval of the work: B.L., N.Y.S., C.H.P., Y.N., S.M.L., K.J.A.

\section{References}

1. Groeschel, S., Chong, W. K., Surtees, R. \& Hanefeld, F. Virchow-Robin spaces on magnetic resonance images: normative data, their dilatation, and a review of the literature. Neuroradiology 48, 745-754, doi:10.1007/s00234-006-0112-1 (2006).

2. Jessen, N. A., Munk, A. S., Lundgaard, I. \& Nedergaard, M. The Glymphatic System: A Beginner's Guide. Neurochemical research 40, 2583-2599, doi:10.1007/s11064-015-1581-6 (2015).

3. Wardlaw, J. M. et al. Neuroimaging standards for research into small vessel disease and its contribution to ageing and neurodegeneration. The Lancet. Neurology 12, 822-838, doi:10.1016/s1474-4422(13)70124-8 (2013).

4. Brown, R. et al. Understanding the role of the perivascular space in cerebral small vessel disease. Cardiovascular research 114, 1462-1473, doi:10.1093/cvr/cvy113 (2018).

5. Doubal, F. N., MacLullich, A. M., Ferguson, K. J., Dennis, M. S. \& Wardlaw, J. M. Enlarged perivascular spaces on MRI are a feature of cerebral small vessel disease. Stroke 41, 450-454, doi:10.1161/strokeaha.109.564914 (2010). 
6. Zhu, Y. C. et al. Severity of dilated Virchow-Robin spaces is associated with age, blood pressure, and MRI markers of small vessel disease: a population-based study. Stroke 41, 2483-2490, doi:10.1161/strokeaha.110.591586 (2010).

7. Duperron, M. G. et al. High dilated perivascular space burden: a new MRI marker for risk of intracerebral hemorrhage. Neurobiology of aging 84, 158-165, doi:10.1016/j.neurobiolaging.2019.08.031 (2019).

8. Achiron, A. \& Faibel, M. Sandlike appearance of Virchow-Robin spaces in early multiple sclerosis: a novel neuroradiologic marker. AJNR. American journal of neuroradiology 23, 376-380 (2002).

9. Wuerfel, J. et al. Perivascular spaces-MRI marker of inflammatory activity in the brain? Brain : a journal of neurology 131, 2332-2340, doi:10.1093/brain/awn171 (2008).

10. Banerjee, G. et al. MRI-visible perivascular space location is associated with Alzheimer's disease independently of amyloid burden. Brain : a journal of neurology $140,1107-1116$, doi:10.1093/brain/awx003 (2017).

11. Park, Y. W. et al. Magnetic Resonance Imaging-Visible Perivascular Spaces in Basal Ganglia Predict Cognitive Decline in Parkinson's Disease. Movement disorders : official journal of the Movement Disorder Society 34, 1672-1679, doi:10.1002/mds.27798 (2019).

12. Mestre, H., Kostrikov, S., Mehta, R. I. \& Nedergaard, M. Perivascular spaces, glymphatic dysfunction, and small vessel disease. Clinical science (London, England : 1979) 131, 2257-2274, doi:10.1042/cs20160381 (2017).

13. Duperron, M. G. et al. Burden of Dilated Perivascular Spaces, an Emerging Marker of Cerebral Small Vessel Disease, Is Highly Heritable. Stroke 49, 282-287, doi:10.1161/strokeaha.117.019309 (2018).

14. Choi, Y. et al. MRI-visible dilated perivascular spaces in healthy young adults: A twin heritability study. Human brain mapping 41, 5313-5324, doi:10.1002/hbm.25194 (2020).

15. Van Essen, D. C. et al. The WU-Minn Human Connectome Project: an overview. Neurolmage 80, 62-79, doi:10.1016/j.neuroimage.2013.05.041 (2013).

16. Wang, Z., Bovik, A. C., Sheikh, H. R. \& Simoncelli, E. P. Image quality assessment: from error visibility to structural similarity. IEEE transactions on image processing : a publication of the IEEE Signal Processing Society 13, 600-612, doi:10.1109/tip.2003.819861 (2004).

17. Dice, L. R. Measures of the amount of ecologic association between species. J Ecology 26, 297-302 (1945).

18. Heaton, R. K. et al. Reliability and validity of composite scores from the NIH Toolbox Cognition Battery in adults. Journal of the International Neuropsychological Society : JINS 20, 588-598, doi:10.1017/s1355617714000241 (2014).

19. Winger, D. G. \& Nason, K. S. Propensity-score analysis in thoracic surgery: When, why, and an introduction to how. The Journal of thoracic and cardiovascular surgery 151, 1484-1487, doi:10.1016/j.jtcvs.2016.02.036 (2016).

20. Mani, M. Twin Study Methods: Intraclass Correlation. (2006). 
21. Sachdev, P. S. et al. White Matter Hyperintensities Are Under Strong Genetic Influence. Stroke 47, 1422-1428, doi:10.1161/strokeaha.116.012532 (2016).

22. Gregg, N. M. et al. Incidental Cerebral Microbleeds and Cerebral Blood Flow in Elderly Individuals. JAMA neurology 72, 1021-1028, doi:10.1001/jamaneurol.2015.1359 (2015).

23. Shi, Y. et al. Cerebral blood flow in small vessel disease: A systematic review and meta-analysis. Journal of cerebral blood flow and metabolism : official journal of the International Society of Cerebral Blood Flow and Metabolism 36, 1653-1667, doi:10.1177/0271678x16662891 (2016).

24. Jiang, J. et al. Cerebral Blood Flow in Community-Based Older Twins Is Moderately Heritable: An Arterial Spin Labeling Perfusion Imaging Study. Frontiers in aging neuroscience 11, 169, doi:10.3389/fnagi.2019.00169 (2019).

25. Martinez-Ramirez, S. et al. Topography of dilated perivascular spaces in subjects from a memory clinic cohort. Neurology 80, 1551-1556, doi:10.1212/WNL.0b013e31828f1876 (2013).

26. Charidimou, A. et al. MRI-visible perivascular spaces in cerebral amyloid angiopathy and hypertensive arteriopathy. Neurology 88, 1157-1164, doi:10.1212/wnl.0000000000003746 (2017).

27. Roher, A. E. et al. Cortical and leptomeningeal cerebrovascular amyloid and white matter pathology in Alzheimer's disease. Molecular medicine (Cambridge, Mass.) 9, 112-122 (2003).

28. Charidimou, A. et al. Enlarged perivascular spaces as a marker of underlying arteriopathy in intracerebral haemorrhage: a multicentre MRI cohort study. Journal of neurology, neurosurgery, and psychiatry 84, 624-629, doi:10.1136/jnnp-2012-304434 (2013).

29. Debette, S., Schilling, S., Duperron, M. G., Larsson, S. C. \& Markus, H. S. Clinical Significance of Magnetic Resonance Imaging Markers of Vascular Brain Injury: A Systematic Review and Metaanalysis. JAMA neurology 76, 81-94, doi:10.1001/jamaneurol.2018.3122 (2019).

30. Zhu, Y. C. et al. High degree of dilated Virchow-Robin spaces on MRI is associated with increased risk of dementia. Journal of Alzheimer's disease : JAD 22, 663-672, doi:10.3233/jad-2010-100378 (2010).

31. Ding, J. et al. Large Perivascular Spaces Visible on Magnetic Resonance Imaging, Cerebral Small Vessel Disease Progression, and Risk of Dementia: The Age, Gene/Environment SusceptibilityReykjavik Study. JAMA neurology 74, 1105-1112, doi:10.1001/jamaneurol.2017.1397 (2017).

32. Lau, K. K. et al. Clinical Correlates, Ethnic Differences, and Prognostic Implications of Perivascular Spaces in Transient Ischemic Attack and Ischemic Stroke. Stroke 48, 1470-1477, doi:10.1161/strokeaha.117.016694 (2017).

33. Boulouis, G. et al. Hemorrhage recurrence risk factors in cerebral amyloid angiopathy: Comparative analysis of the overall small vessel disease severity score versus individual neuroimaging markers. Journal of the neurological sciences 380, 64-67, doi:10.1016/j.jns.2017.07.015 (2017).

34. Shen, T. et al. The role of brain perivascular space burden in early-stage Parkinson's disease. NPJ Parkinson's disease 7, 12, doi:10.1038/s41531-021-00155-0 (2021).

35. Fénelon, G., Gray, F., Wallays, C., Poirier, J. \& Guillard, A. Parkinsonism and dilatation of the perivascular spaces (état criblé) of the striatum: a clinical, magnetic resonance imaging, and 
pathological study. Movement disorders : official journal of the Movement Disorder Society 10, 754760, doi:10.1002/mds.870100609 (1995).

36. Lampe, L. et al. Lesion location matters: The relationships between white matter hyperintensities on cognition in the healthy elderly. Journal of cerebral blood flow and metabolism : official journal of the International Society of Cerebral Blood Flow and Metabolism 39, 36-43, doi:10.1177/0271678x17740501 (2019).

37. Brugulat-Serrat, A. et al. Patterns of white matter hyperintensities associated with cognition in middle-aged cognitively healthy individuals. Brain imaging and behavior 14, 2012-2023, doi:10.1007/s11682-019-00151-2 (2020).

\section{Figures}

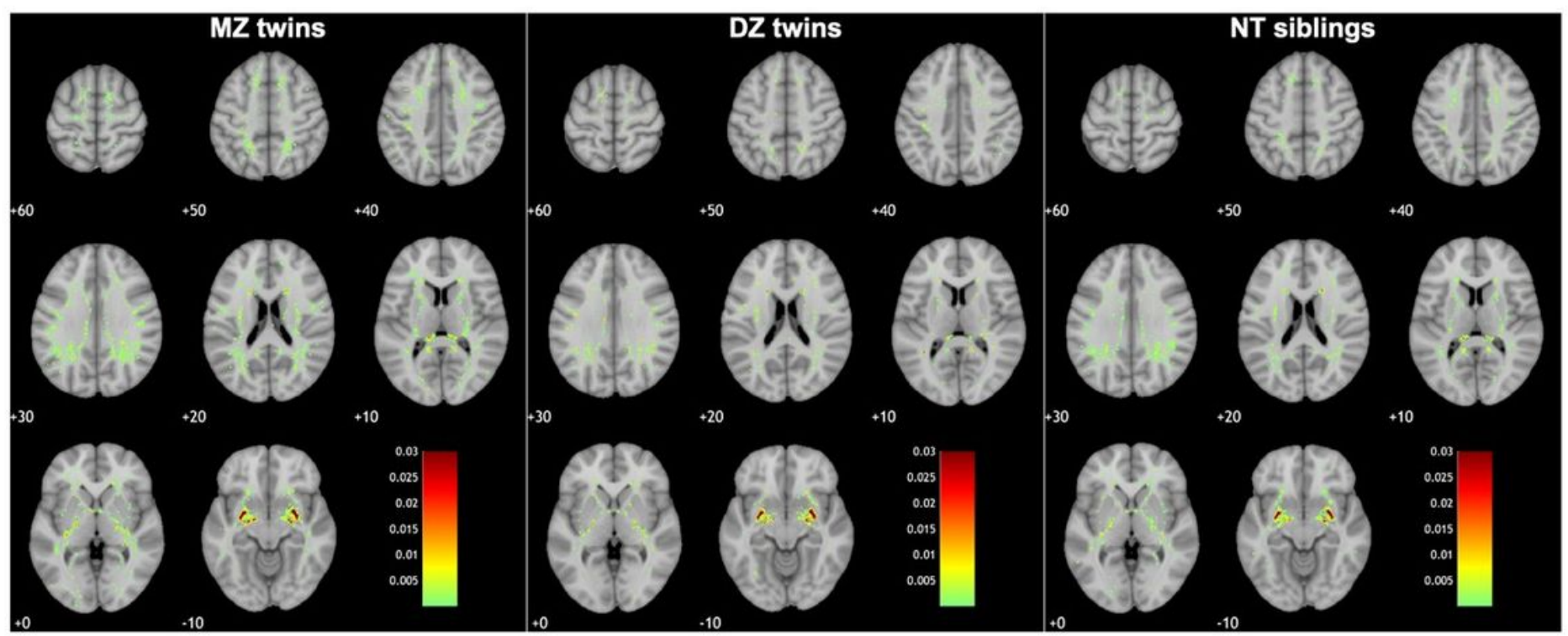

\section{Figure 1}

Likelihood map of overlapping dPVS voxels between paired subjects across all subjects for each group

\section{Supplementary Files}

This is a list of supplementary files associated with this preprint. Click to download.

- SupplementaryTable.docx 